\title{
Padrões de reflectância foliar de eucalipto em diferentes dias e períodos do dia
}

\author{
Luiz Felipe Ramalho de Oliveira, Reynaldo Campos Santana
}

Universidade Federal dos Vales do Jequitinhonha e Mucuri - UFVJM. E-mail: luizfelipe@florestal.eng.br

\section{Resumo}

Diante da complexa relação entre reflectância foliar e aspectos fisiológicos da planta em dias e diferentes horários do dia, a pesquisa foi desenvolvida para averiguar e mensurar o grau da influência do dia e o período do dia em espectros de reflectância foliar de árvores de eucalipto. 0 experimento foi conduzido em um plantio de eucalipto clonal, híbrido espontâneo de Eucalyptus grandis com centroide nas coordenadas

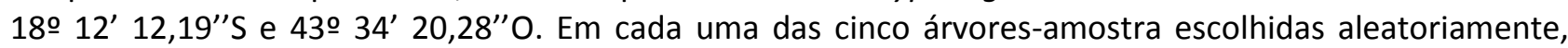
amostraram-se quatro folhas distribuídas em cada ponto cardeal. Todas as folhas selecionadas foram avaliadas nos dias 7, 8, 9 e 10 de dezembro de 2013, em dois períodos do dia: entre 8 e 9 horas e entre 15 e 16 horas. A leitura da reflectância foliar foi realizada utilizando um mini-espectrometro com posterior suavização e dessa forma realizou-se a análise de componentes principais e com posterior análise de agrupamento por dendrograma. No geral, a média das reflectâncias foliares no segundo dia foram as menores e as médias do terceiro dia maiores. Em média a reflectância foliar foi menor no período da manhã do que no período da tarde. O dia e o período do dia em que foi realizada a leitura influenciaram de forma aleatória na reflectância foliar de eucalipto. A reflectância foliar do eucalipto pode se alterar em um curto período de tempo, inclusive em um mesmo dia, o que indica a importância da padronização no horário de coleta para futuros estudos.

Palavras-chave: agrupamento; espectro eletromagnético; PCA; variação diária.

\section{Eucalyptus leaf reflectance patterns on different days and periods of the day}

\begin{abstract}
Faced with the complex relationship between leaf reflectance and physiological aspects of the plant on days and at different times of the day, the research was developed to ascertain and measure the influence of day and period of the day on leaf reflectance of eucalyptus trees. The experiment was carried out in a clonal eucalyptus stand, spontaneous Eucalyptus grandis hybrid with centroid at 18ㅇ 12 '12,19'S and 43은 20,28 "W. In each of the five sample trees randomly selected, four leaves were sampled at each cardinal point. All selected leaves were evaluated on days 7, 8, 9 and 10 of December 2013, in two periods of the day: between 8 and 9 hours and between 15 and 16 hours. The leaf reflectance was measured using a minispectrometer with subsequent smoothing and the analysis of principal components and subsequent analysis of dendrogram cluster were performed. In general, the average leaf reflectance on the second day was the lowest and the third day average was higher. Leaf reflectance was lower in the morning than in the afternoon. The day and period of the day of reading were randomly influenced by eucalypt leaf reflectance. The leaf reflectance of eucalyptus can change in a short time, even in the same day, which indicates the importance of the standardization in the collection schedule for future studies.
\end{abstract}

Keywords: cluster; electromagnetic spectrum; PCA; daily changes.

\section{Introdução}

As árvores em plantios de eucalipto
passam por diferentes estádios de

desenvolvimento. Durante seu ciclo de cultivo acontecem variações das concentrações de componentes bioquímicos e propriedades 
estruturais das suas folhas. Nos últimos anos, a relação destas propriedades químicas e estruturais e a reflectância foliar vem sendo estudadas em diferentes espécies, variações de tempo, condições ambientais e experimentais (GATES et al., 1965; GITELSON et al., 2009; USTIN et al., 2009; OLIVEIRA et al., 2017).

A reflectância foliar é a relação entre a energia eletromagnética incidente em uma folha e a energia que é refletida pela mesma. Sua utilização vem se destacando e ganhando cada vez mais espaço no manejo de espécies florestais e agrícolas (GITELSON et al., 2009, USTIN et al., 2009; GAMON et al., 2015; OLIVEIRA et al., 2017). Por ser uma técnica não-destrutiva, torna-se possível monitorar estresses hídricos, deficiências nutricionais e várias outras características fisiológicas das plantas de forma rápida e precisa (DECHANT et al., 2017; OLIVEIRA et al., 2017).

Geralmente, as regiões do espectro eletromagnético mais utilizadas para análise da reflectância foliar são a do visível (VIS, $400-700$ $\mathrm{nm}$ ) e infravermelho próximo (NIR, $701-2500$ $\mathrm{nm})$. Isso porque, nessas regiões a energia eletromagnética interage com pigmentos foliares, responsáveis por absorver o espectro luminoso, e com estruturas foliares (GATES et al., 1965). Avanços em pesquisas relacionadas a reflectância foliar e concentração de compostos bioquímicos vem sendo obtidos em espécies florestais, sobretudo para espécies de eucalipto (DATT et al., 1998; 1999; SIMS; GAMON, 2002; OLIVEIRA et al., 2017).

Contudo, estes trabalhos têm se restringindo na avaliação de partes pequenas do espectro de reflectância, principalmente com uso de índices de vegetação, e uma avaliação contínua do espectro de reflectância nas regiões do VIS-NIR não são completamente abordadas (USTIN et al., 2009; OLLINGER, 2011). Apesar disso, índices de vegetação vem sendo utilizados para identificação de diversos compostos bioquímicos, como teores de nutrientes e pigmentos foliares, taxa te transporte de elétrons e velocidade máxima de carboxilação da Rubisco (PIMSTEIN et al., 2011; DECHANT et al., 2017; OLIVEIRA et al., 2017), bem como para captar variações sazonais e diárias em plantas durante diferentes estádios fenológicos de produção (GAMON et al., 1992; SOUZA et al., 2009; SUSZECK et al., 2010; GAMON et al., 2015).

A utilização de um espectro contínuo de reflectância apresenta as vantagens de se obter maiores informações em uma única amostra.
Desta forma, torna-se possível captar mais precisamente alterações no espectro de reflectância foliar de uma planta submetida a algum estresse. Haja vista que esse estresse pode ocasionar alterações em diversas regiões do espectro eletromagnético. Portanto, os índices de vegetação, que são compostos por apenas pequenas partes do espectro eletromagnético, podem não identificar as alterações na reflectância foliar de uma planta sob estresse e assim, não atestar a real situação fisiológica da planta.

Diante da complexa relação entre aspectos fisiológicos da planta e reflectância foliar coletada em dias e horários do dia distintos, a pesquisa foi desenvolvida para averiguar e mensurar o grau da influência do dia e o período do dia em espectros de reflectância foliar de árvores de eucalipto em dias chuvosos.

\section{Material e Métodos}

O experimento foi conduzido em um plantio de eucalipto clonal, híbrido espontâneo de Eucalyptus grandis (I-144) aos 12 meses de idade, implantado no arranjo $12 \times 2 \mathrm{~m}$ em Neossolo quartzarênico (EMBRAPA, 2013), com centroide nas coordenadas 18 $12^{\prime} 12,19^{\prime \prime} \mathrm{S}$ e 43 ㅇ $34^{\prime} 20,28^{\prime \prime} O$, no município de Diamantina, Minas Gerais - Brasil.

Como o trabalho pauta-se na identificação da influência do dia e horário na coleta da refletância foliar, optou-se por amostrar folhas localizadas no terço basal das copas das árvores. Esta região foi escolhida por possuir uma ciclagem de nutrientes mais intensa, - que proporciona uma maior atividade fisiológica e consequente maior possibilidade de se identificar a influência do dia e horário na reflectância foliar (SAUR et al., 2000).

Com o intuito de descartar a possível influência do ângulo da radiação solar nas folhas, em cada uma das cinco árvores-amostra $(A 1, A 2$, A3, A4 e A5) escolhidas aleatoriamente, amostraram-se quatro folhas distribuídas em cada ponto cardeal. Todas as folhas selecionadas foram avaliadas nos dias 7 (D1), 8 (D2), 9 (D3) e 10 (D4) de dezembro de 2013, em dois períodos do dia: entre 8 e 9 horas (P1) e entre 15 e 16 horas (P2). Devido a intensa chuva e ventania no terceiro dia, não foi possível realizar a leitura no período da manhã (Figura 1). Os dados de temperatura média diária foram obtidos por meio do data logger 88128-AZ e a precipitação diária por meio do pluviômetro automático 
modelo TE525-series, ambos instalados a $10 \mathrm{~m}$ do

local do experimento.

Figura 1. Precipitação e temperatura média diária durante o período experimental na área do experimento (M= Manhã; $\mathrm{T}=$ Tarde).

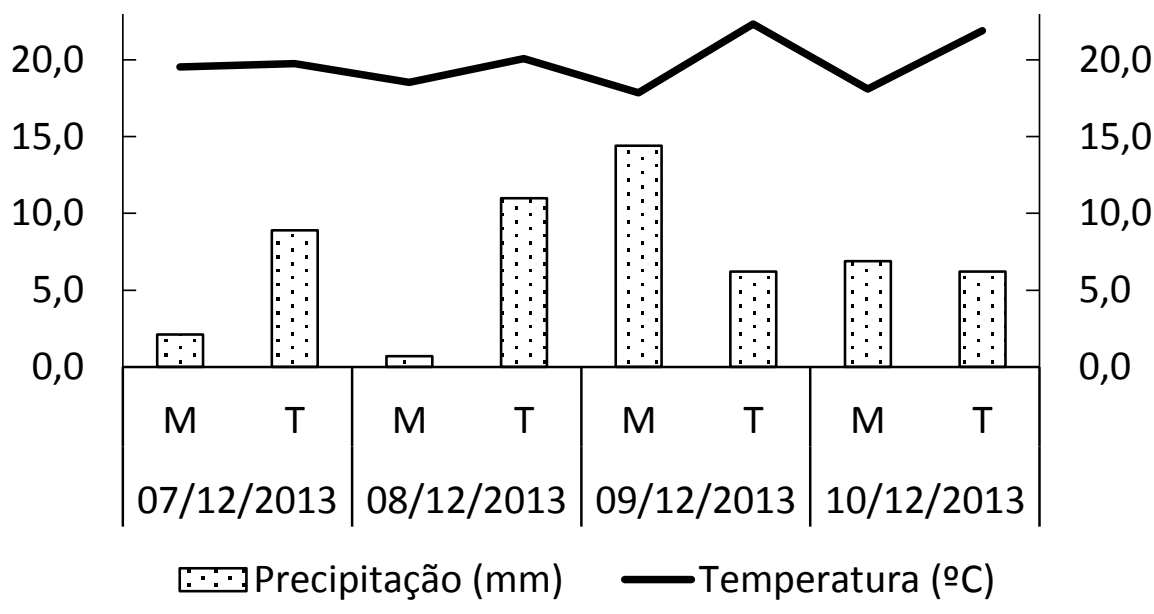

A leitura da reflectância foliar (400-900 $\mathrm{nm}$ ) foi realizada na parte abaxial de cada folha utilizando o mini-espectrometro $\mathrm{Cl}$-710 (CID BIOSCIENCE - Camas, Washington, EUA) e analisada pelo SpectraSnap! (software versão 1.1.3.150, CID Bio-Science), com tempo de integração de 300 milissegundos, largura de suavização de 10 pontos com média a cada duas leituras.

Posteriormente, a reflectância foliar foi suavizada utilizando o algoritmo Savistky-Golay (SAVITZKY; GOLAY, 1964) com grau de polinômio dois. Do conjunto de 4 espectros suavizados avaliados em cada árvore-amostra por dia e período, realizou-se a média e desta forma foi obtida uma reflectância foliar por árvore-amostra em cada dia e período. No total foram obtidas 35 amostras para análises.

Com a reflectância foliar suavizada foi realizada a análise de componentes principais (PCA) no intuito de reduzir a dimensionalidade dos dados, analisar a influência dos dias e horários na reflectância foliar das árvores e visualizar tendências de agrupamento das amostras. A PCA reduz a dimensionalidade dos dados gerando novas variáveis pela combinação linear das variáveis iniciais. A PCA apresenta estas novas variáveis em uma dimensão $k$ sempre menor do que aquela de dimensão $d$ (número de comprimentos de onda presentes no espetro de reflectância foliar). O conjunto de novas dimensões gerados são chamados de Componentes Principais (PCs) e cada PC é direcionado a máxima variância.

Após a PCA, foram analisados o gráfico da variância máxima explicada pelos PCs e os gráficos de dispersão. Posteriormente, procedeuse a análise de agrupamento por um dendrograma, utilizando o método completo com a distância euclidiana definindo os valores do espaço entre os vizinhos mais próximos e avaliando a extensão e direção dos afastamentos entre os valores médios das variáveis na discriminação dos grupos.

\section{Resultados e Discussão}

Diferentes árvores apresentaram reflectâncias foliares distintas (Figura 2). Em média, a A1 apresentou menor reflectância foliar na região do VIS e a A2 apresentou maior reflectância nessa região. Menores valores de reflectância na região do VIS indicam uma árvore em melhores condições fisiológicas, com maiores teores de clorofila e nitrogênio (GITELSON et al., 2009, USTIN et al., 2009; OLIVEIRA et al., 2017). Enquanto que maiores valores de reflectância foliar nesta região indicam o oposto. 
Figura 2. Médias e desvios padrões da reflectância foliar. A. Em cada árvore avaliada; B. Em cada dia do experimento (D3: reflectância apenas do período da tarde); C. Em cada período de avaliação.
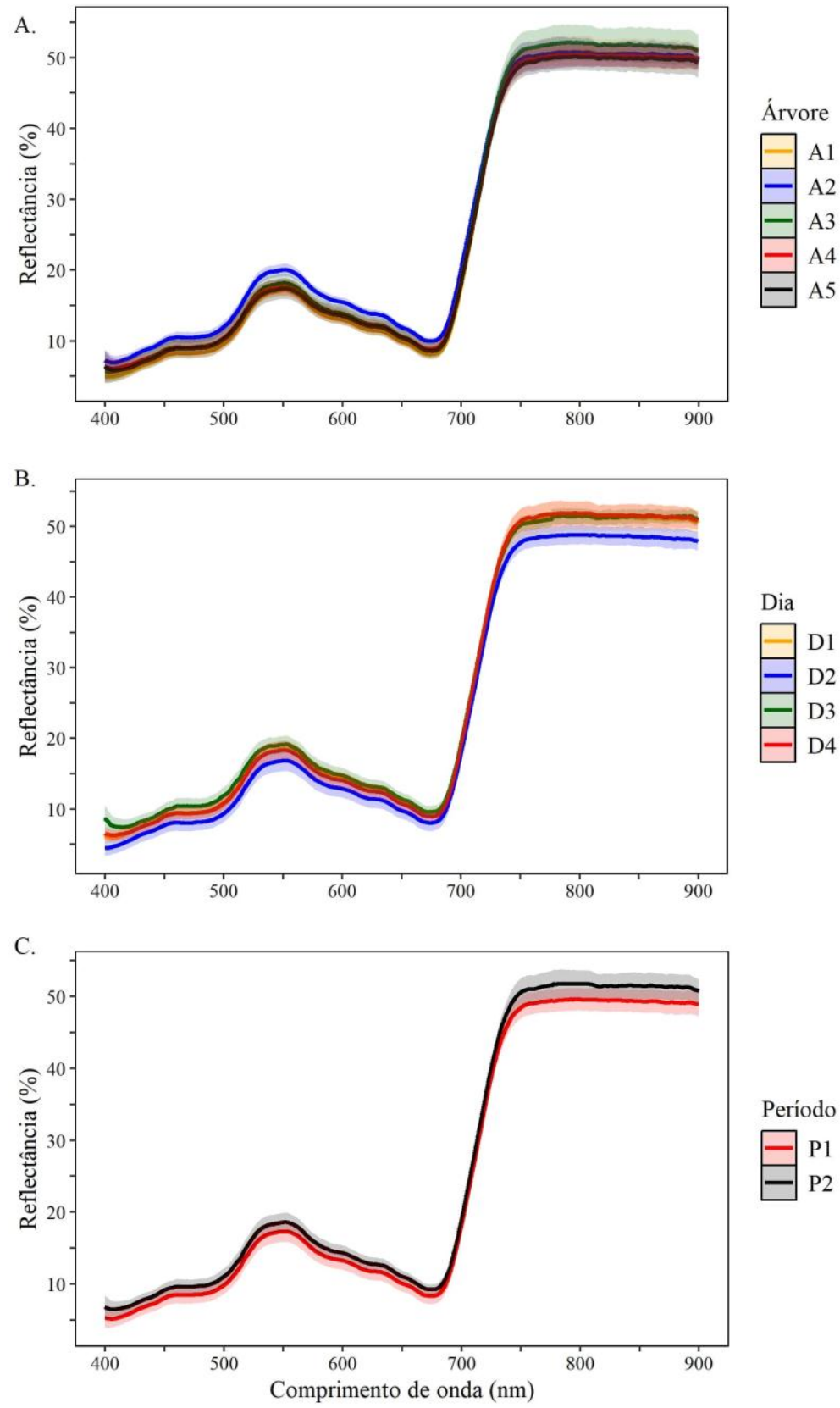

No geral, a média das reflectâncias foliares no D2 foram as menores e as médias do D3 maiores (Figura 2 B.). Como discutido anteriormente, este fato indica que no D2, as árvores estavam em melhores condições fisiológicas (RICHARDSON et al., 2002; OLIVEIRA et al., 2017; DECHANT et al., 2017). Enquanto que no D3, dia com maiores precipitações, as árvores estavam em condições fisiológicas adversas (Figura 2 B.). Alterações diárias da reflectância foliar geralmente são mais facilmente observadas quando há o processo de senescência foliar ou quando são avaliados períodos de tempo mais amplos (HATFIELD et al., 2008, DILLEN et al., 2012; GAMON et al., 2015). No entanto, como pode ser verificado neste estudo, existe uma variação na reflectância foliar em pequenos intervalos de tempo.

Em relação ao horário de coleta de reflectância, em média a reflectância foliar foi menor no período da manhã do que no período da tarde (Figura 2 C.). Indicando que, durante os dias de estudo, o período da manhã foi o de maior atividade fisiológica para o Eucalipto. Sabese que a reflectância foliar oscila com a elevação do sol, atingindo seu valor máximo entorno das 12 horas, no entanto ao amanhecer e ao pôr-dosol essa reflectância apresenta valores semelhantes. Em trabalho pioneiro, Gamon et al. (1992) verificou a maior atividade fotossintética 
de plantas de girassol no período da manhã utilizando o índice de reflectância fotoquímico (PRI). Assim como, Souza et al. (2009) e Suszeck et al. (2010), também utilizando índices de vegetação, verificaram maior atividade fotossintética do milho no período de manhã (8-9 h) do que a tarde (15-16 h). Bem como, Gamon et al. (2015) verificaram que a atividade fotossintética em duas espécies de pinus foi maior no período da manhã do que a tarde. Isso porque diferentes taxas fotossintéticas ocorrem durante o dia alterando o metabolismo foliar, assim como situações de estresse também podem ocasionar mudanças na reflectância foliar (RUNNING; NEMANI, 1988; DAVIS, 1995).

Pela PCA, as 10 PCs acumularam 99,53\% da variância total, dos quais $80,97 \%$ da variância máxima foi explicada pelo PC1, 16,25\% explicada pelo PC2, 1,67\% pelo PC3, 0,42\% pelo PC4, 0,28\% pelo $\mathrm{PC} 5,0,09 \%$ pelo $\mathrm{PC} 6,0,07 \%$ pelo $\mathrm{PC} 7,0,04 \%$ pelo PC8, 0,03\% pelo PC9 e 0,02\% pelo PC10 (Figura 3).

Figura 3. Variância máxima explicada pelos 10 primeiros componentes principais.

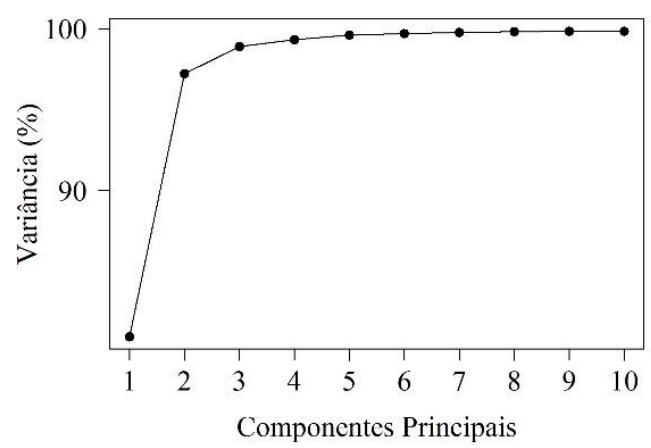

Apenas para a A3 é possível notar uma tendência de agrupamento nos diferentes dias e horários (Figura $4 \mathrm{~A}$.). As demais árvores foram dispersas no gráfico sem apresentar um padrão de agrupamento. Analisando este mesmo gráfico, mas com sua coloração destacando o dia de avaliação (Figura 4 B.), verifica-se que as reflectâncias foliares no D2 foram as que apresentaram uma maior tendência de agrupamento. Não obstante, foi neste dia em que a média das reflectâncias foliares se apresentaram em menor porcentagem, indicando uma maior padronização da atividade fotossintética destas árvores no plantio (Figura 2 B.). Quando analisado o período de coleta da reflectância foliar, verifica-se uma tendência de agrupamento no período da manhã e tarde, desencadeado principalmente pelo PC1 (Figura 2 c.). 
Figura 4. Gráfico de dispersão entre os dois primeiros componentes principais (PC1 x PC2). A. Amostras da reflectância foliar coloridas por árvore; B. Amostras da reflectância foliar coloridas por dia de avaliação; C. Amostras coloridas pelo período do dia em que houve a coleta da reflectância foliar.
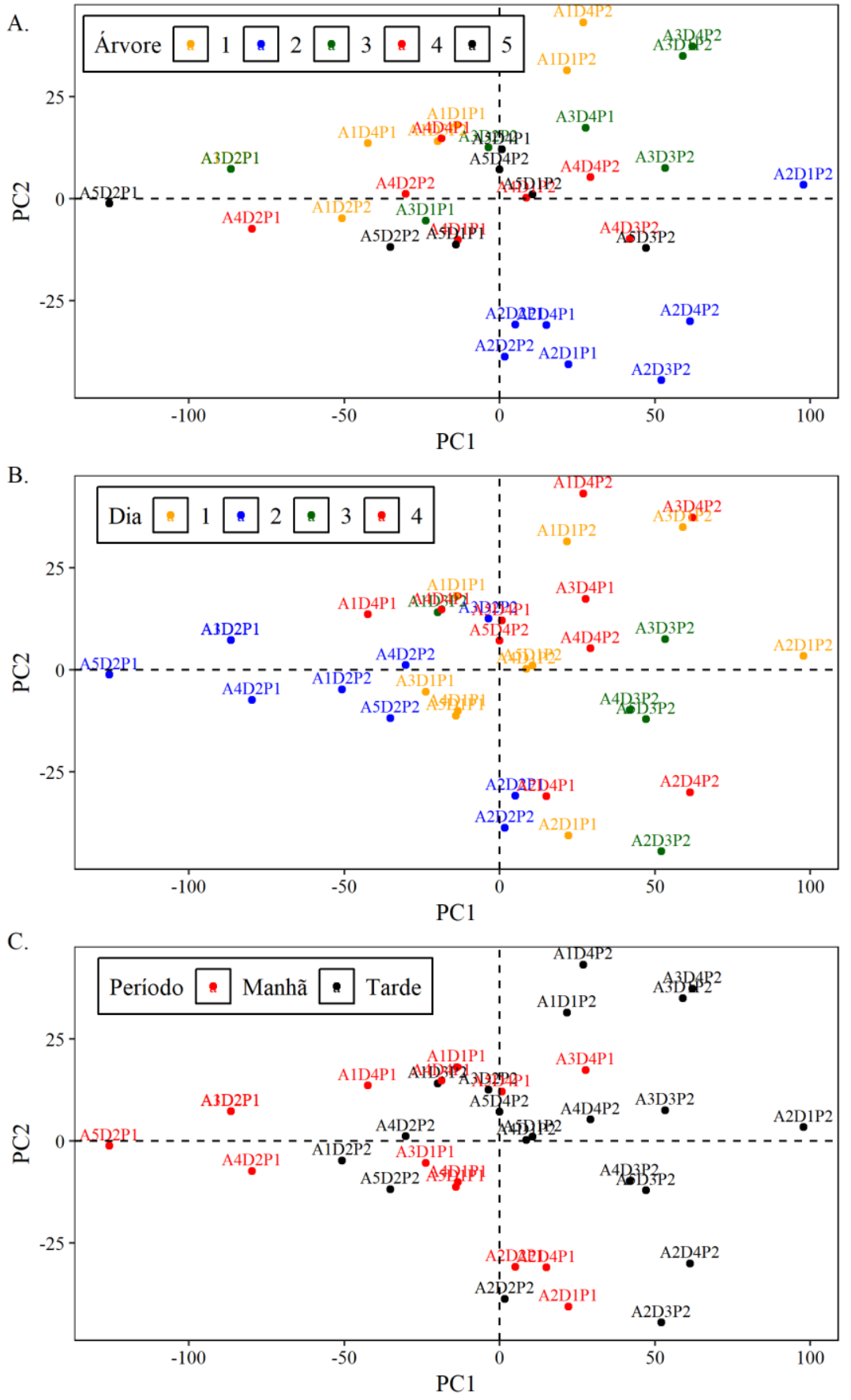

No entanto, toda a tendência de agrupamento verificada no gráfico de dispersão da PCA foi aleatória e apenas o período de coleta da reflectância foliar segue uma tendência de agrupamento entre manhã e tarde (Figura 4).
Fato confirmado ao analisar o agrupamento pelo dendrograma, o qual mostrou que os dias e períodos do dia influenciam na reflectância de maneira aleatória (Figura 5). 
Figura 5. Dendrograma de agrupamento da reflectância foliar.

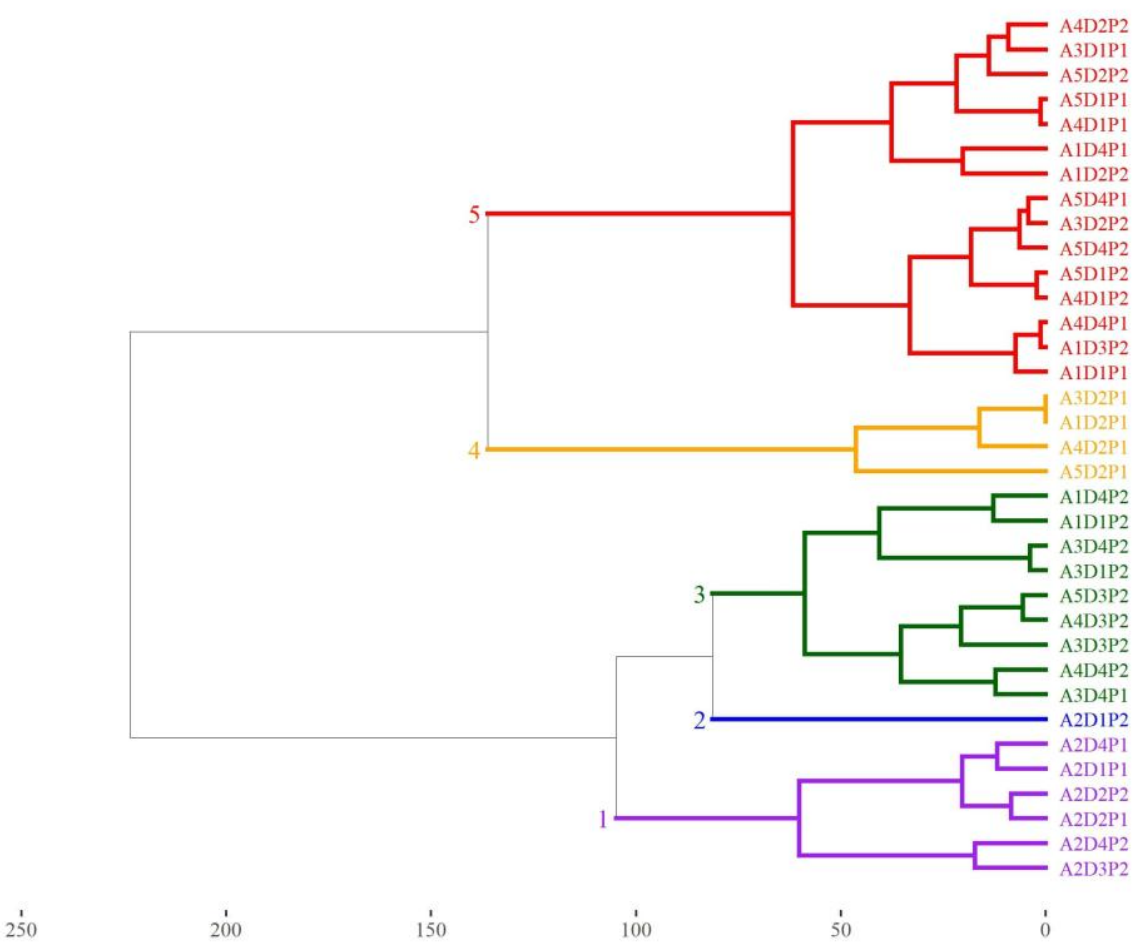

Apenas para a $\mathrm{A} 2$, estas variáveis não apresentaram grande influência (Figura 4). Isso porque apenas uma amostra da reflectância foliar dessa árvore não se enquadrou no grupo 1 , composto apenas pela reflectância foliar deste indivíduo em diferentes dias e períodos. $\mathrm{O}$ grupo 2 foi composto pela reflectância foliar da A2 que não se enquadrou no grupo 1 . Com exceção da reflectância foliar A3D4P1, o grupo 3 foi composto por reflectâncias foliares coletadas no P2. O Grupo 4 foi formado apenas por reflectâncias foliares coletadas no D2P1. Enquanto que o grupo 5 foi composto por reflectâncias foliares de diferentes árvores, dias e períodos de coleta.

Além da influência da elevação solar na reflectância foliar, estresses hídricos são capazes de alterar a reflectância foliar em questão de horas (SUN et al., 2008). Como o experimento foi realizado em período chuvoso, as altas precipitações podem ter sido um fator relevante para as alterações abruptas da reflectância foliar das árvores em dias e períodos distintos (Figura 1, 2).

\section{Conclusões}

Os dias e períodos do dia influenciaram de forma aleatória na reflectância foliar de eucalipto. A reflectância foliar do eucalipto pode se alterar em um curto período de tempo, inclusive em um mesmo dia, o que indica a importância da padronização no horário de coleta para futuros estudos. Desta forma, sugere-se que a coleta da reflectância foliar seja realizada sempre em um mesmo período do dia, ou pela manhã ou apenas no período da tarde. Além disso, sugere-se a realização de mais estudos em períodos de estiagem, para que seja verificada se há um padrão de alteração na reflectância foliar das árvores de eucalipto.

\section{Agradecimentos}

O presente trabalho foi realizado com apoio da Coordenação de Aperfeiçoamento de Pessoal de Nível Superior - Brasil (CAPES) - Código de Financiamento 001, agradecemos também o apoio da Universidade Federal dos Vales do Jequitinhonha e Mucuri -UFVJM, Conselho Nacional de Desenvolvimento Científico e Tecnológico - CNPq, Fundação de Amparo à Pesquisa de Minas Gerais - FAPEMIG.

\section{Referências}

DAVIS, J. G. Impact of time of day and time since irrigation on cotton leaf blade and petiole nutrient concentrations. Communications in Soil Science and Plant Analysis, v. 26, p. 2351-2360, 1995.

https://doi.org/10.1080/00103629509369452

DECHANT, B.; CUNTZ, M.; VOHLAND, M.; SCHULZ, E.; DOKTOR, D. Estimation of photosynthesis 
traits from leaf reflectance spectra: Correlation to nitrogen concentration as the dominat mechanism. Remote Sensing of Environment, v. 196, p.279-292, 2017. http://dx.doi.org/10.1016/j.rse.2017.05.019

DILLEN, S. Y.; BEECK, M. O.; HUFKENS, K.; BUONANDUCI, M.; PHILLIPS, N. G. Seasonal patterns of foliar reflectance in relation to photosynthetic capacity and color index in two co-occurring trees species, Quercus rubra and Betula papyrifera. Agricultural and Forest Meteorology, v.160, p.60-68, 2012. https://doi.org/10.1016/j.agrformet.2012.03.001

EMBRAPA. Sistema brasileiro de classificação de solos. Brasília: Embrapa, 2013.

GAMON, J. A.; PEÑUELAS, J.; FIELD, C. B. A narrow-waveband spectral index that tracks diurnal changes in photosynthetic efficiency. Remote Sensing of Environment, v. 41, p. 35-44, 1992.

https://doi.org/10.1016/0034-

4257(92)90059-S

GAMON, J. A.; KOVALCHUCK, O.; WONG, C. Y. S.; HARRIS, A.; GARRITY, S. R. Monitoring seasonal and diurnal changes in photosynthetic pigments with automated PRI and NDVI sensors. Biogeosciences, v.12, p.4149-4159, 2015. https://doi.org/10.5194/bg-12-4149-2015

GITELSON, A. A.; CHIVKUNOVA, O. B.; MERZLYAK, M. N. Nondestructive estimation of anthocyanins and chlorophylls in anthocyanic leaves. American Journal of Botany, v. 96, n. 10, p. 1861-1868, 2009. http://dx.doi.org/10.3732/ajb.0800395

HATFIELD, J. L.; GITELSON, A. A.; SCHEPERS, J. S.; WALTHALL, C. L. Application of spectral remote sensing for agronomic decisions. Agronomy Journal, v.1, p.117-131, 2008. http://dx.doi.org/10.2134/agronj2006.0370c

OLIVEIRA, L. F. R.; OLIVEIRA, M. L. R.; GOMES, F. S.; SANTANA, R. C. Estimating foliar nitrogen in Eucalyptus using vegetation indexes. Scientia Agricola, v.74, n.2, p.142-147, 2017. http://dx.doi.org/10.1590/1678-992X-2015-0477.

OLLINGER, S. V. Sources of variability in canopy reflectance and the convergent properties of plants. New Phytologist, v.189, p.375-394, 2011. https://doi.org/10.1111/j.1469-

8137.2010.03536.x

RICHARDSON, A. D.; DUIGAN, S. P.; BERLYN, G. P. An evaluation of noninvasive methods to estimate foliar chlorophyll content. New Phytologist, v.153, p.185-194, 2002. https://doi.org/10.1046/i.0028646X.2001.00289.X

RUNNING, S. R.; NEMANI, R. Season patterns of the reflectance indices, carotenoid pigments and photosynthesis of evergreen chaparral species. Oecologia, v.131, p.366-374, 1988. https://doi.org/10.1007/s00442-002-0905-9

SAUR, E., NAMBIAR, E. K. S., FIFE, D. N. Foliar nutrient retranslocation in Eucalyptus globulus. Tree Physiology, v. 20, n. 16, p. 1105-1112, 2000. https://doi.org/10.1093/treephys/20.16.1105

SOUZA, E. G.; ROCHA, T.; URIBE-OPAZO, M. A.; NÓBREGA, L. H. P. Índices de vegetação no milho em função da hora do dia e da taxa de nitrogênio aplicada. Revista Brasileira de Engenharia Agrícola e Ambiental, v.13, p.865-872, 2009. https://doi.org/10.1590/S1415$\underline{43662009000700008}$

SUN, P.; GRIGNETTI, A.; LIU, S.; CASACCHIA, R.; SALVATORI, R.; PIETRINI, F.; LORETO, F.; CENTRITTO, $M$. Associated changes in physiological parameters and spectral reflectance indices in olive (Olea europaea L.) leaves in response to different levels of water stress. International Journal of Remote Sensing, v. 29, n.6, p.1725-1743, 2008. https://doi.org/10.1080/01431160701373754

SUSZECK, G.; SOUZA, E. G.; URIBE-OPAZO, M. A.; NÓBREGA, L. H.; KONOPAZKI, R. S. Influência da direção da semeadura e da hora do dia em índices de vegetação. Engenharia na Agricultura, v.18, n.5, p.405-412, 2010. https://doi.org/10.13083/reveng.v18i5.250

USTIN, S. L.; GITELSON, A. A.; JACQUEMOUD, S.; SCHAEPMAN, M.; ASNER, G. P.; GAMON, J. A.; ZARCO-TEJADA, P. Retrivial of foliar information about plant pigment systems from high resolution spectroscopy. Remote Sensing of Environment, v.113, p.567-577, 2009. https://doi.org/10.1016/i.rse.2008.10.019 